\title{
LA CONSTRUCCIÓN DE LA IDENTIDAD NACIONAL A TRAVÉS DE LOS CUADERNOS ESCOLARES EN EL FRANQUISMO EN EL PAÍS VASCO ${ }^{1}$
}

\author{
Pauli Dávila Balsera \\ Universidad del País Vasco/Euskal Herriko Unibertsitatea UPV/EHU/España \\ pauli.davila@ehu.eus \\ Luis M. Naya Garmendia \\ Universidad del País Vasco/Euskal Herriko Unibertsitatea UPV/EHU/España \\ luisma.naya@ehu.eus
}

\begin{abstract}
RESUMEN
Los cuadernos escolares son un buen indicador para conocer el currículum prescrito. En el caso del País Vasco durante el franquismo nos encontramos con dos situaciones claramente contrapuestas, mientras que en las escuelas nacionales se seguía un curriculum donde aparece claramente explícita la ideología franquista, denominada en el primer periodo nacionalcatólica, en el campo de la resistencia semiclandestina de algunas poblaciones podemos observar que los cuadernos muestran una visión nacionalista desde la perspectiva del nacionalismo vasco. Por lo tanto, se trata de una situación donde el curriculum en las diferentes instituciones está impregnado por dos tipos de nacionalismo: el español y el vasco. En un campo de confrontación nacional, es evidente que el Estado contó con toda sus agentes para imponer su ideología, mientras que en el campo de la resistencia era más débil esta construcción nacional. En los dos casos, la escuela y los cuadernos son un buen agente de transmisión ideológica. En este trabajo presentamos el análisis de una pequeña parte del fondo de cuadernos que está recogido en el Museo de la Educación de la Universidad del País Vasco, centrándonos más en los cuadernos de las primeras ikastolas ya que no han sido analizados hasta el momento.
\end{abstract}

Palabras clave: Cuadernos Escolares. País Vasco. Identidad. Franquismo. Ikastolas.

\section{THE CONSTRUCTION OF NATIONAL IDENTITY THROUGH SCHOOL NOTEBOOKS DURING THE FRANCOISM IN THE BASQUE COUNTRY}

\begin{abstract}
School notebooks are a good indicator to know the prescribed curriculum. In the case of the Basque Country, during the Francoism, we find two contrasting situations. Whereas in the national schools, where a curriculum explicitly based on Franco's ideology was thought, in the semi-secrecy of some towns we can observe that the notebooks show a nationalist vision from a Basque nationalism perspective. Therefore, the same curriculum was impregnated by two types of nationalism: the Spanish and the Basque one. In a moment of national confrontation, it is clear that the State counted on all its staff to impose its ideology, while in the resistance field there was a weaker national construction. In both cases, the school and the

\footnotetext{
${ }^{1}$ Esta aportación es resultado de un proyecto de investigación financiado por el Ministerio de Economía y Competitividad, proyecto número EDU2013-44129-P. Los autores son miembros del Grupo de Estudios Históricos y Comparados en Educación - Garaian, reconocido por el Gobierno Vasco con el número IT 603-13 y de la Unidad de Formación e Investigación "Educación, Cultura y Sociedad (UFI 11/54)" de la Universidad del País Vasco/Euskal Herriko Unibertsitatea UPV/EHU.
} 
notebooks were a good agent of ideological transmission. In this work we present the analysis of a little part of the notebook stockage that is gathered up at the Museum of Education of the University of the Basque Country. We focus on the notebooks belonging to the first Ikastolas since they have not been analysed up to this moment.

Keywords: School Notebooks. Basque Country. Identity. Francoism. Ikastolas.

\section{INTRODUCCIÓN}

El exilio, tanto interior como exterior, producido en el País Vasco como consecuencia de la finalización de la guerra civil española, afectó a todos los ámbitos de la vida cotidiana, al igual que en el resto del Estado. Pero, mientras que en la mayoría de las provincias el proyecto educativo franquista triunfaba bajo la denominación del nacional-catolicismo, el hecho diferencial de la lengua y la cultura agravará la situación en el País Vasco, aplicándose con mayor rigor las disposiciones sobre la censura o la depuración del magisterio, sobre todo en Guipúzcoa y Vizcaya. Todo aquello que fuese sospechoso de ir en contra de la unidad de la patria o de la propagación de ideas disolventes verá una respuesta inmediata: obligar a bautizar a los niños con nombres en castellano, renombrar los barcos que tuviesen nombres en euskera o prohibir hablar en público en euskera (EUSKALTZAINDIA, 1977). La desaparición de cualquier elemento que recordase la pervivencia de las experiencias educativas de la República y la expansión del modelo educativo español no son más que dos caras de un mismo proceso de homogeneización del sistema educativo llevado a cabo por el franquismo. En este sentido, los cuadernos de la época recogen perfectamente las claves de la ideología franquista en dos aspectos relevantes: la unidad de la patria y la presencia de la religión en todos los ámbitos.

En el contexto de la primera etapa del franquismo, surgirá una experiencia singular de escolarización infantil a cargo de una maestra nacionalista Elbira Zipitria (1906-1982) (EUSKERAZAINTZA, 1986) que volverá de su exilio en Francia en la década de los años cuarenta. Así, a partir de 1946, surgen las primeras "etxe eskolas" (escuelas domesticas) en San Sebastián. La experiencia educativa puesta en marcha por Elbira Zipitria (ARANBURU, 1991) será imitada por otras maestras, discípulas suyas, en el periodo entre 1946 a 1960, si bien la ilustre pedagoga continuaría con su propia escuela hasta 1968. Esta experiencia es una muestra de la resistencia educativa contra el sistema educativo español en un periodo donde la inevitable clandestinidad daba cobertura a experiencias educativas aisladas. Estas escuelas acogían a una escasa población infantil vascoparlante que, obligatoriamente, para finalizar su 
escolarización debían recurrir a las escuelas nacionales españolas al cumplir los nueve años (GARMENDIA; ETXEZARRETA, 2009).

La experiencia educativa de Elbira Zipitria debe insertarse en lo que hemos denominado alfabetización restringida (DÁVILA, 1995) en tanto que su objetivo es una alfabetización dirigida a los vascoparlantes, con escaso número de castellanoparlantes, que aprenderán el euskera de forma autodidacta. En este sentido, la alfabetización infantil fue importante y comenzó a desarrollarse a partir de actividades de lectura en estas primeras escuelas vascas. El uso del euskera en esta época, dada la represión existente, se mantendrá en un nivel privado y en contextos sociales restringidos. En un nivel más académico, hacia el final del período, se darán los pasos más importantes para la estandarización lingüística del euskera (1968), que dará lugar al euskera batua (euskara unificado) y que supondrá en las etapas siguientes una forma de normativización lingüística que, paulatinamente, se introducirá en las ikastolas y será adoptado mayoritariamente como norma tanto para textos literarios como científicos. El euskera batua suponía relegar los dialectos existentes, al menos en su aspecto escrito. Este fenómeno no fue del agradado de Elbira Zipitria que defendía la utilización del euskera dialectal. Un aspecto que caracteriza este modelo de alfabetización es, precisamente, la permanencia del código ideológico nacionalista que continuará manteniendo, de forma soterrada, las elaboraciones ideológicas de los seguidores de Sabino Arana o del nacionalismo tradicional en un contexto de represión. Desde un punto de vista ideológico se puede constatar la permanencia de valores como la religión y la raza en el código nacionalista. No obstante, este nacionalismo puede calificarse de residual, si tenemos en consideración el surgimiento de otros planteamientos tanto culturales como políticos que intentan sintonizar con un nacionalismo más radical, sobre todo a partir de la década de los sesenta. El surgimiento de las primeras ikastolas irá poniendo las bases de la escolarización y, consecuentemente, sentando la posibilidad de una alfabetización de la población infantil, que en este período será mayoritariamente vascoparlante.

Como sea que los estudios sobre el franquismo son suficientemente conocidos (MAYORDOMO; FERNÁNDEZ, 1993) y en cierta medida, también los análisis de los libros de texto para el caso vasco (BILBAO; EZKURDIA; PEREZ, 2005) y los cuadernos escolares (MARTÍN; RAMOS, 2015), por lo que respecta a los cuadernos escolares de los que disponemos en el Museo de la Educación de la Universidad del País Vasco, encontramos imágenes que plasman el curriculum del primer franquismo, con una clara presencia de símbolos con una clara representación nacional española, religiosa y con un culto a la figura 
de los soldados que están luchando en el frente. Estos símbolos no son solamente gráficos, sino que también impregnan, incluso los análisis sintácticos de las frases.

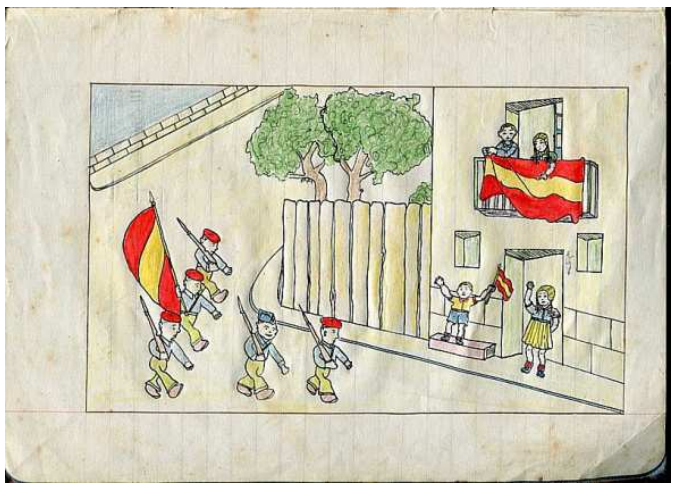

Fuente: Sancho, C. (1938), cuaderno escolar depositado en el Museo de la Educación de la Universidad del País Vasco, Signatura EK 48, p. 4.

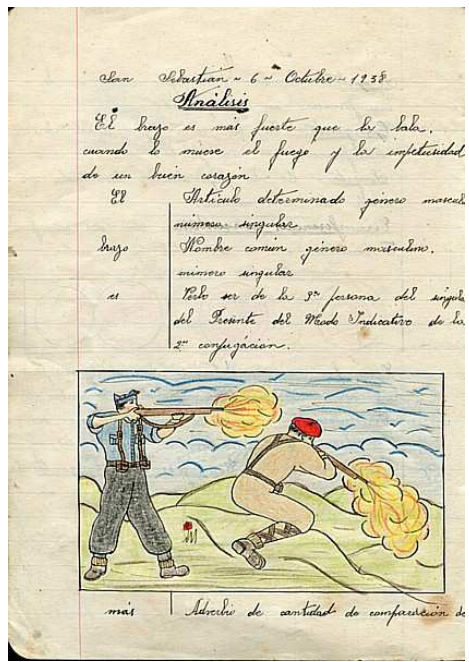

Fuente: Sancho, C. (1938), cuaderno escolar depositado en el Museo de la Educación de la Universidad del País Vasco, Signatura EK 48, p. 8.

En contraste con estos cuadernos, en los que de manera explica aparece la ideología nacional católica en el País Vasco al igual que en el resto del Estado, los cuadernos de las primeras escuelas vascas recurren al mismo mecanismo de trasmisión ideológica, pero desde la perspectiva del nacionalismo vasco, en un contexto de represión cultural y lingüística.

\section{LA PEDAGOGÍA DE ELBIRA ZIPITRIA}

Para poder estudiar los planteamientos pedagógicos de Elbira Zipitria no podemos recurrir a ningún texto pedagógico propio de la autora, ya que no dejó obra escrita. No obstante, sus seguidoras nos han legado una exhaustiva información de su biografía y prácticas pedagógicas (ABÁSOLO, LÓPEZ DE MUNIAN, 2004; ARANBURU, 1992). A partir del conocimiento que tenemos de sus prácticas podemos conocer sus planteamientos educativos, ideológicos y de innovación pedagógica.

Elbira Zipitira, a lo largo de su vida, fue una persona muy ligada al Partido Nacionalista Vasco y a Emakume Abertzale Batza [Unión de Mujeres Patriotas]. Durante la República tuvo un importante papel como oradora en muchos mítines del partido, así como vinculación a la defensa de la educación, el euskera, etc., y del papel de la mujer en el campo de la política activa. A la vuelta del exilio puso en marcha una experiencia educativa 
desarrollada en su casa particular, sita en la Parte Vieja de Donostia, denominada etxe-eskola (enseñanza doméstica), cuya duración se prolongó por más de veinte años. Algunos historiadores de las ikastolas han preferido denominar a esta etapa como "clandestina", ya que en los primeros periodos el acceso a la casa particular se llevaba de una forma irregular. A finales de los años 60, con la puesta en marcha de la "cartilla de escolaridad" y del movimiento de ikastolas, las etxe-eskolas comienzan a cerrar o a transformarse en ikastolas, dando lugar a una tercera red, además de las escuelas privada y pública (BASURKO, 1995).

En cuanto a sus aportaciones pedagógicas, las que tiene un reconocimiento mayor con respecto al curriculum son las relativas a la enseñanza de las matemáticas y del euskara. No obstante, tal y como desarrollaba las clases (las salidas al entorno, la introducción de cantos, la recogida de material de la propia naturaleza, etc.) podemos afirmar que llevaba a cabo una enseñanza activa, siguiendo unas fuentes pedagógicas que son claramente reconocibles en Montessori, Décroly, Piaget o Freinet. En este sentido, algunos libros conservados en su biblioteca muestran que tenía conocimiento de los planteamientos de la Escuela Nueva, así como de las prácticas educativas innovadoras desarrolladas en Francia (ABÁSOLO; LÓPEZ DE MUNIAN, 2004). Además de estos conocimientos pedagógicos hemos de tener presente que, a la vista de los cuadernos que hemos analizado, se aprecia que en el curriculum impartido, la religión tiene una presencia importante, así como algunos contenidos nacionalistas. Estos últimos proceden del libro de Ixaka Lopez Mendizabal Xabiertxo que, como señala Karmele Esnal, una de sus seguidoras, era utilizado habitualmente. Además de este libro utilizaba otros materiales como las canicas, palillos, lápices bicolores (rojo y azul) y blocs de dibujo (ARANBURU, 1991).

El Xabiertxo, cuya primera edición es de 1923, es un icono que compendia todos los conocimientos que debían adquirirse en la escuela primaria; casi podríamos afirmar que se trata de la primera enciclopedia que sintetiza el curriculum vasco y en el que predominan la religión, la familia y las obligaciones de los niños y el conocimiento del medio, sobre todo el ambiente rural (FERNÁNDEZ, 1995). A pesar de ser un texto previo a la Segunda República su uso fue muy extendido, no solo en las etxe-eskolas, sino posteriormente en las ikastolas. De este libro se han impreso varias ediciones, alguna de ella en Buenos Aires (1943), donde se exilió el autor. La última es de 2003, lo cual es un indicador de su éxito. Casi podría decirse que no hay niño/a euskaldun que no lo haya utilizado de una u otra manera, guardando en la memoria un grato recuerdo del mismo por su sencillez, su valor comunicativo y el acompañamiento de dibujos muy adelantados para su época. 


\section{CUESTIONES METODOLÓGICAS}

El análisis de los cuadernos escolares es muy rico y existen diversas aproximaciones interesantes para el caso español (MEDA y otros, 2010; MARTíN; RAMOS, 2013, 2015). No obstante, el análisis de las imágenes de dichos cuadernos está menos explotado. En la obra de Meda, Montino y Sani, tan sólo hemos podido encontrar un trabajo que analiza las imágenes (FERNÁNDEZ; WELTI; BISELLI, 2010), además del trabajo posterior de Meda (2012). También puede encontrarse una síntesis interesante de la cuestión en otros trabajos del mismo autor (MEDA, 2014). Un conjunto interesante de trabajos sobre análisis de dibujos fue llevado a cabo por el Grupo de Investigación de Vic y, asimismo, en las Jornades d'Història de l'Educació celebradas en 2014 en Palma de Mallorca (Colleldemont, 2014).

Los cuadernos que analizamos son una parte del fondo que está recogido en el Museo de la Educación de la Universidad del País Vasco. Los correspondientes a la etxe-eskola de Elbira Zipitria, provienen de la donación de una de sus alumnas, que en 2012 dejó en depósito en el mismo un total de 37 cuadernos escolares, realizados tanto por ella como por su hermano durante los años 1958 a 1968. Los cuadernos comienzan cuando uno de los autores tenía cinco años y sorprende encontrar algún cuaderno de cuando tenía catorce años. La explicación a este hecho es que el alumno continuaba su escolarización siguiendo el sistema dual anterior a la Ley General de Educación de 1970, mientras que la hermana pasaría a cursar el bachillerato elemental, graduándose posteriormente en magisterio y pedagogía en la Universidad Pontificia de Salamanca. En cuanto a la función que cumplían estos cuadernos en la etxe-eskola de Elbira Zipitria es evidente que se trata de trabajos y dibujos que obedecen al mandato recibido por parte de la maestra. Los cuadernos de la etapa franquista provienen asimismo de donaciones y depósitos recibidos en el Museo de la Educación.

Respecto a los cuadernos de la etxe-eskola que hemos analizado, tenemos que señalar que no hay una continuidad temporal, pero que la mayoría están realizados en los primeros años de la década de los sesenta y se corresponden, en su mayoría, a dos alumnos con edades comprendidas entre los 6 y los 9 años. Los cuadernos utilizados no eran los cuadernos escolares habituales comercializados por empresas productoras de los mismos (Rubio o similares), sino que eran cuadernos comerciales destinados a realizar dibujo lineal o dibujo libre (en algunos casos de las empresas Balandro o Aris). Los cuadernos son de cuarenta páginas de 242 x $170 \mathrm{~mm}$., y están encuadernados con una espiral metálica. El gramaje del papel es de 200, lo que permitía que los alumnos desarrollaran diversas tareas sobre una superficie que aguantaba muy bien los inseguros trazos infantiles. Los alumnos utilizaban 
para escribir un lápiz bicolor azul y rojo, muy típicos en esa época, aunque posteriormente se constata la utilización de bolígrafos y lápices de color negro. Un elemento común en todos esto cuadernos es que en la primera página aparece una exaltación de la oración Gloria: Aitaren eta Semearen eta Espiritu Santuaren izenean. Aintza Aitari eta Semeari eta Espiritu Santuari. Asieran zen bezalaxe orain eta beti gizaldi eta gizaldietan [En el nombre del Padre, del Hijo y del Espíritu Santo. Gloria al Padre, al Hijo y al Espíritu Santo. Como era en un principio, ahora y siempre, por los siglos de los siglos].

Los correspondientes al primer franquismo están escritos en cuadernos comerciales de hojas grapadas, están, también, profusamente ilustrados y cubren años diferentes.

\section{LOS DIBUJOS EN LOS CUADERNOS ESCOLARES DEL ALUMNADO DE ELBIRA ZIPITRIA}

El análisis que hemos realizado sobre estos cuadernos se centra en la iconografía y los dibujos que figuran en los mismos. Para ello hemos clasificado algunos de los dibujos de los cuadernos en función de la presencia de las dimensiones: Identidad vasca y entorno y contexto local.

\section{Identidad Vasca}

Los cuadernos que estamos analizando transmiten no solamente el curriculum previsto para un alumnado de edad inferior a los nueve años, sino también una forma de entender el entorno en el que estos vivían. Como sabemos, el alumnado que frecuentaba la etxe-eskola de Elbira Zipitria procedía, en general, de un entorno nacionalista y tenía el euskara como lengua materna. Fiel a los principios nacionalistas, Elbira no hubiera aceptado, previsiblemente, un alumno cuya lengua materna no fuese el euskera ya que el mismo Sabino Arana construyó su nacionalismo en base a una población vasca, entendida con un cierto sentido de raza. Por lo tanto, cabe pensar que, tanto las familias, como Elbira Zipitria y sus discípulas compartían un ideario común, perteneciente a lo que hemos denominado como nacionalismo residual.

Dentro de este marco interpretativo cabe analizar el contenido de vasquidad subyacente en los dibujos de los cuadernos escolares. Nos parece más pertinente hablar de vasquidad y no de nacionalismo ya que en el contexto social y político en el cual se desarrollan estas etxe-eskola, no podían expresarse de forma explícita los valores que componían el nacionalismo vasco. No obstante, sí que hemos podido apreciar que los dibujos 
transmiten muchos elementos de identidad que permiten entender la voluntad de configurar unos contenidos acordes con la ideología nacionalista. Hemos de tener en cuenta que en estos años el Partido Nacionalista Vasco estaba en el exilio y no será hasta una década más tarde cuando los exiliados comiencen a volver al País Vasco, principalmente de América Latina y Francia. Por lo tanto, si bien de manera explícita no podemos configurar el universo nacionalista, sí que podemos registrar una serie de temas que configurarían una forma de entender Euzkadi (siguiendo la nomenclatura de Sabino Arana). Para ello hemos elaborado tres subcategorías en las cuales podríamos incluir una serie de temas que conformarían ese bricolaje nacionalista: fiestas populares y folklore; símbolos y paisaje y representaciones del territorio. Es decir, se trata de elementos que vinculan al alumnado con el universo nacionalista, a pesar de que se recurra a diversos tipos de representaciones.

\section{Fiestas populares y folklore}

Con respecto a las fiestas populares y el floklore, en los cuadernos, se hace hincapié en recoger fiestas que se celebran en el entorno local de San Sebastián y que, tradicionalmente, se han ido manteniendo a pesar de que el algún momento se prohibiesen, como fuera el caso de los Carnavales. Todo ello permitía unas relaciones comunitarias a través de esta expresión festiva. Así podemos encontrar dibujos que representan la tamborrada de San Sebastián, los coros de Santa Agueda, bailes vascos o canciones populares.

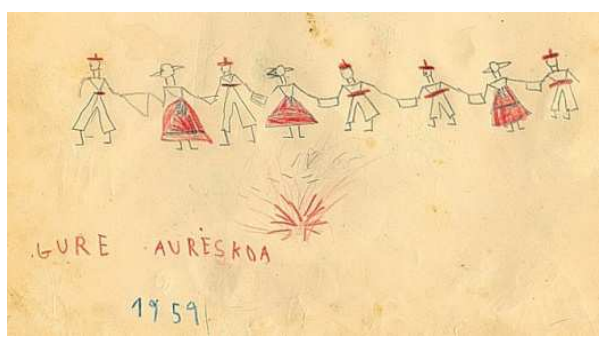

Fuente: Muñoz, X. (1959), cuaderno escolar depositado en el Museo de la Educación de la Universidad del País Vasco, Signatura EK 24, p. 22.

Hay que tener en cuenta que, dentro del nacionalismo español, el fomento del folklore regional era una forma de reivindicar la unidad de España en su diversidad. Sólo desde esta perspectiva puede entenderse la supervivencia de este tipo de manifestaciones festivas que compartían tanto los nacionalistas vascos como el más rancio nacionalismo español, por supuesto, salvando las distancias en lo referente a la diferente significación de cada una de estas manifestaciones. Siguiendo los planteamientos pedagógicos de Elbira, la elaboración de 
estos dibujos coincidiría con la celebración de la fiesta en concreto, como hemos podido observar al ver, en algún caso, la fecha escrita en el dibujo.

\section{Símbolos}

Como hemos señalado, los símbolos claramente nacionalistas, como por ejemplo la ikurriña, no están presentes en los cuadernos. No obstante, sí que hay dos elementos claramente reivindicativos del nacionalismo vasco. Por una parte, la celebración del Aberri Eguna (día de la patria vasca) que se recuerda cada año utilizando simbología muy similar: las montañas del País Vasco con hogueras en sus cumbres llamando a la celebración del día. En la parte inferior del dibujo suele recogerse el año y lugar de convocatoria del mismo. Todo ello con todas las precauciones que suponía en el franquismo este tipo de manifestaciones. Por lo tanto, podríamos entender que hay una manera explícita, en este caso, de convocar a la asistencia al día máximo de la celebración del nacionalismo vasco (el Domingo de Resurrección, siguiendo la propuesta de Sabino Arana).

Otro elemento que nos parece significativo de la simbología más explícita que aparece en los cuadernos es el uso de las estelas funerarias en las que se cita el nombre de personajes del nacionalismo vasco (Sabino Arana y sus seguidores, Lendakari Agirre, etc.) acompañando el dibujo de la siguiente frase: Emaiezu jauna betiko atsedena [Dales Señor el descanso eterno]. No obstante, se aprecia que no solamente se hacían estos dibujos con los personajes citados, sino que se aprovechaba también para rendir homenaje póstumo a personas que hubieran fallecido, bien en el entorno familiar de los niños (familiares de los alumnos, de las profesoras, etc.), bien en el entorno político internacional (Martin Luther King). En esta última, realizada el año de su asesinato (1968) hace mención expresa a su calidad de pacifista, modificando ligeramente la frase clásica ya mencionada. 


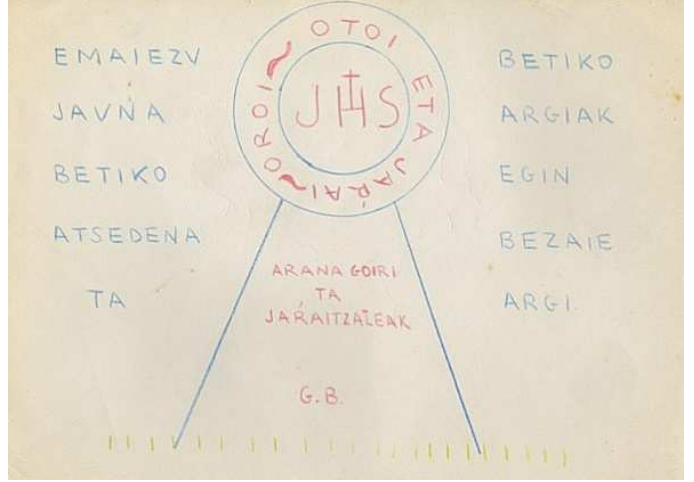

Fuente: Muñoz, X. (1968), cuaderno escolar depositado en el Museo de la Educación de la Universidad del País Vasco, Signatura EK 9, p. 4.

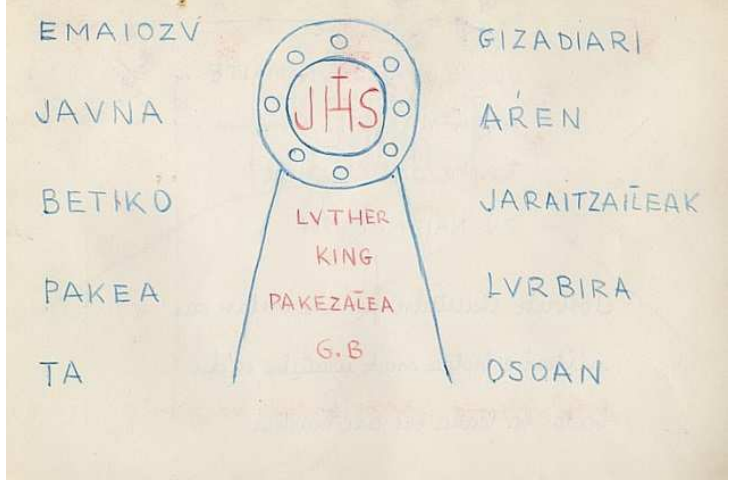

Fuente: Muñoz, X. (1968), cuaderno escolar depositado en el Museo de la Educación de la Universidad del País Vasco, Signatura EK 9, p. 22.

\section{Paisaje y representaciones del territorio}

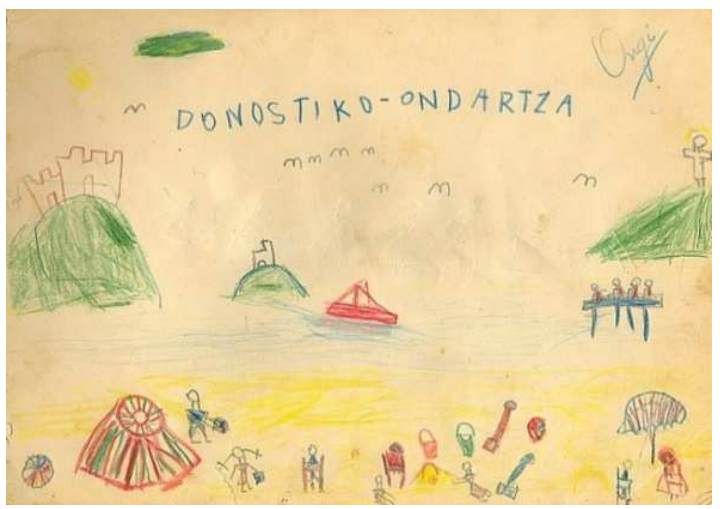

Fuente: Muñoz, X. (1965), cuaderno escolar depositado en el Museo de la Educación de la Universidad del País Vasco, Signatura EK 24, p. 21.

El paisaje representado en los cuadernos está relacionado con la identidad más próxima o local. En este sentido, entendemos que Elbira Zipitria utiliza estos dibujos no solamente para conformar una identidad local, sino para facilitar el aprendizaje, por ejemplo, de los puntos geográficos más conocidos de la ciudad de Donostia. Por lo tanto, estos dibujos cumplen una doble función ya que, en algunos casos, aparece el dibujo del incomparable marco de la Bahía de Donostia, en un sentido lúdico y veraniego, recogiendo niños en la playa, veleros y barcos pesqueros, etc.; y en otros, de una manera más esquemática, esa misma representación sirve para enseñar los accidentes geográficos más próximos-

Donde aparece claramente una identificación nacionalista, sin lugar a dudas es en la representación del mapa de Euskal Herria, incluyendo las siete provincias vascas, prescindiendo de la frontera franco-española. Este mapa aparece en numerosas ocasiones, aunque tenemos que señalar que, en la mayoría de los casos, se trata de una copia fidedigna del dibujo que aparece en el Xabiertxo. No podemos olvidar que los mapas de España que 
figuran en las escuelas nacionales representan las provincias españolas y sus regiones, y que el País Vasco, siempre aparece bajo el epígrafe de "Provincias Vascongadas", separando Navarra y no mencionando, por supuesto, los territorios situados en el Estado francés. Al igual que en el caso de la representación gráfica de Donostia, también en este caso se utiliza, en algunas ocasiones, para señalar los accidentes geográficos del territorio. El mapa de Euskal Herria, en la denominación de Euzkadi es un elemento de identificación nacional y de unidad de los patriotas vascos. Por lo tanto, al igual que hacía la escuela nacional y, en general, cualquier otro tipo de nacionalismo el mapa propio es la mejor forma gráfica de identidad territorial, razón por la cual Elbira Zipitria no pudiese soslayar su utilización. Hay que recordar que ya en un algún texto anterior a la guerra civil se recurría al mapa de Euskal Herria, haciendo alusión a una hipotética forma de corazón para fomentar la identificación emocional de los niños con el territorio (DÁVILA, 2008).

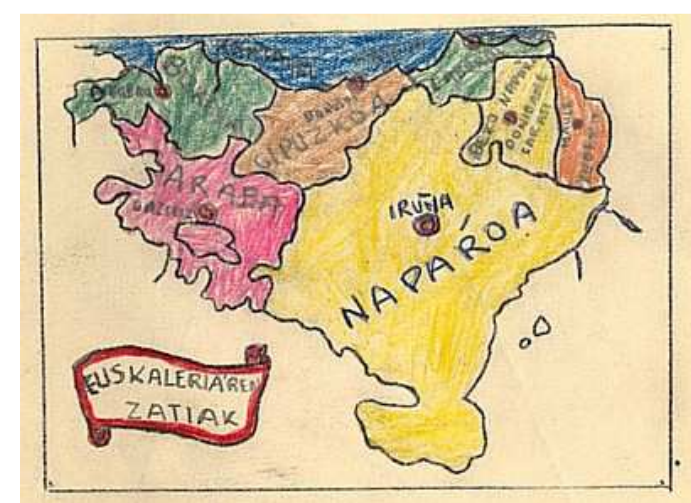

Fuente: Muñoz, X. (1965), cuaderno escolar depositado en el Museo de la Educación de la Universidad del País Vasco, Signatura EK 6, p. 20.

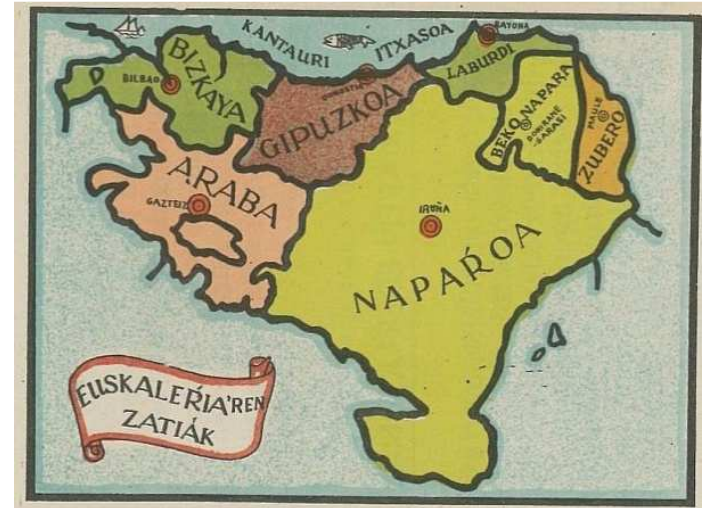

Fuente: López Mendizabal, I (1923), Xabiertxo, Tolosa, López Mendizabal, p. 100.

Al margen de estas representaciones de Euskal Herria sorprende un ejercicio geográfico en el cual se pretende mostrar Euzkadi en el mundo a través de un globo terráqueo y un texto en castellano. El alarde de vascocentrismo no deja de ser ingenuo y típico, por otra parte, de cualquier construcción nacionalista, y a la vez muestra las características de un texto tópicamente nacionalista, al comenzar diciendo que "Nosotros vivimos en una tierra que se llama Euzkadi. Otros viven en las tierras de alrededor". No obstante, hay que leerlo también en su vertiente pedagógica, tanto por su filosofía de pedagogía activa como el objetivo de facilitar el aprendizaje de la gramática española. En el texto aparecen los verbos subrayados en rojo y el resto de partes de la gramática con diferentes marcas, tanto en color como en forma, para luego ser, previsiblemente, la base de un análisis morfológico. Por lo tanto, nuevamente se aprecia la doble función con la que Elbira Zipitria utilizaba este tipo de 
recursos: por una parte, transmitir un mensaje de vasquidad y, por otra, utilizar un recurso pedagógico para el aprendizaje de diversas materias. No obstante, hay que tener en cuenta que siendo la religión un elemento importantísimo dentro de ideología nacionalista, todo lo dicho sobre identidad vasca, debe complementarse con lo relativo a la religión ya que es una idea fundamental dentro de la ideología nacionalista. Elbira Zipitria conoció el nacionalismo de preguerra y, por lo tanto, participaba de los conceptos sabinianos sobre el nacionalismo. Por lo tanto, es fácil imaginar que en la etxe-eskola que regentaba los contenidos religiosos tendrían una importante presencia. Como apuntaba una de sus discípulas, $\mathbf{M}^{\mathrm{a}}$ Karmen Mitxelena, al referirse a la filosofía pedagógica de Zipitria, señalaba "Egia esan, Elbirak erlijioari garrantzi ematen zion eta nik uste, baita ikasleen gurasoek ere [A decir verdad, Elbira daba importancia a la religión y, en mi opinión, también los padres de los alumnos]" (ARAMBURU, 1991, p. 49). Por lo tanto, cabe pensar que esta situación se reflejara en sus cuadernos.

\section{Entorno y Contexto Local}

Como hemos podido observar en el apartado relativo a la identidad vasca y a la religión, el recurso de Elbira Zipitria era muy ilustrativo en tanto que la representación de estos valores estaba mediatizada por actividades que el alumnado conocía en su propia experiencia cotidiana. Sus conocimientos de pedagogía activa facilitaban este tipo de abordaje en el aprendizaje de diversas materias. Esta forma de plantearse la enseñanza está mucho más presente en los trabajos donde los niños y niñas dibujan escenas de la naturaleza o de las actividades productivas del entorno. Es el caso del conocimiento de las estaciones del año, la pesca o el trabajo en el caserío.

No podemos ignorar que Elbira Zipitria acostumbraba a pasear con sus alumnos por dos emplazamientos muy cercanos a donde estaba situada su etxe-eskola: el monte Urgull y la bahía y el puerto donostiarras. Por lo tanto, los dibujos en los que se recogen las estaciones, el paisaje de la bahía o la descarga del pescado en el puerto eran escenas que los alumnos conocían por experiencia directa. En este sentido se puede apreciar ciertas escenas que pueden ser categorizadas como "dibujo libre" y otras donde se ve claramente que son una copia del libro Xabiertxo, no solo en los aspectos de imagen, sino también del texto que la acompaña. En este sentido, en la etxe-eskola se seguía una práctica extendida en cuanto a la escritura en los cuadernos escolares (DEL POZO; RAMOS, 2010). 
EGUBALDI ONA

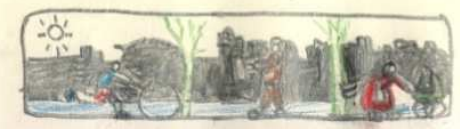

Fuente: Muñoz, X. (1963), cuaderno escolar depositado en el Museo de la Educación de la Universidad del País Vasco, Registro EK 18, p. 39.

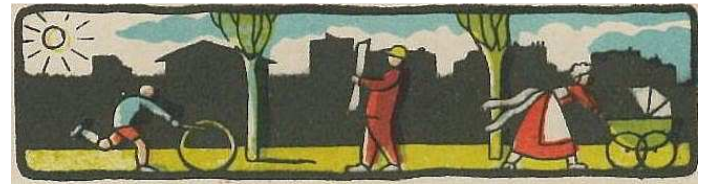

Fuente: López Mendizabal, I (1923), Xabiertxo, Tolosa, Lopez Mendizabal, p. 82.

\section{CONCLUSIONES}

Los cuadernos escolares son un buen indicador del currículum prescrito. En el caso del país Vasco nos encontramos con dos situaciones contrapuestas. Mientras que en las escuelas nacionales se seguía un curriculum donde aparece claramente explícita la ideología franquista, denominada en el primer periodo nacional-católica, en el campo de la resistencia semiclandestina de algunas poblaciones podemos observar que los cuadernos muestran una visión nacionalista desde la perspectiva del nacionalismo vasco. Por lo tanto, se trata de una situación donde el curriculum en las diferentes instituciones está impregnado por dos tipos de nacionalismo: el español y el vasco. En un campo de confrontación nacional, es evidente que el Estado contó con toda sus agentes para imponer su ideología, mientras que en el campo de la resistencia era más débil esta construcción nacional. En los dos casos, la escuela y los cuadernos son un buen agente de transmisión ideológica. En este trabajo nos hemos centrado más en el caso de las primeras ikastolas.

Durante el franquismo en Donostia-San Sebastián se desarrolló una experiencia educativa (1946-1968) llevada a cabo por la maestra nacionalista Elbira Zipitria en su domicilio particular que fue denominada etxe-eskola (escuela doméstica). Esta escuela era frecuentada por hijos de familia nacionalistas que ya tenían conocimientos de euskera. La obtención de un conjunto de cuadernos escolares utilizados por alumnos que acudieron a dicha escuela nos ha permitido analizar el contenido de los mismos.

El trabajo que hemos realizado se ha centrado en los aspectos iconográficos, sobre todo en los dibujos. Para ello hemos establecido unas dimensiones y unas categorías de análisis que nos han permitido conocer el curriculum impartido en dicha escuela. Los elementos más destacables de este curriculum son la enseñanza de las matemáticas mediante métodos pedagógicos activos, la presencia de la religión y del catecismo, siguiendo los presupuestos nacionalistas al respecto, el entorno local como elemento conocedor del medio y el dibujo libre. 
A pesar de estos métodos de pedagogía activa, hemos podido constatar el uso de modelos de dibujo procedentes principalmente de dos fuentes: por lo que respecta a la enseñanza del medio, el Xabiertxo En ambos casos se aprecia que, tanto en el contenido literario como iconográfico, fueron utilizados siguiendo una práctica muy extendida en el resto de escuelas, donde la copia de un modelo era práctica habitual.

\section{BIBLIOGRAFÍA}

ABÁSOLO, R. y LÓPEZ DE MUNIAN, J. Ilunetik argira. Elbira Zipitria. Donostia: Xangorin, 2004, 140p.

ARANBURU, X. Elbira Zipitria Irastorza, Auñamendi Eusko Entziklopedia, 1992, Disponível em: <http://www.euskomedia.org/aunamendi/147407/129533>. Acceso em: 15 jul. 2015.

Hezkuntzaren historia Euskal Herrian: ikastola. Elbira Zipitriaren biografiarako jakingaiak: ikastola Donostia 1942-1969 urte bitartean. Cuadernos de Sección. Educación. Eusko Ikaskuntza, v. 5, p. 9-74, 1991.

BASURKO, F. La normalización de la ikastola: breve historia y estado de la cuestión de la escuela pública vasca. Lengua, escuela y cultura. El proceso de alfabetización en Euskal Herria, siglos XIX y XX. Leoia, Universidad del País Vasco, 1995, p. 193-220.

BILBAO, B.; EZKURDIA, G. y PEREZ, K. Basque textbooks during the 20th century: the content and the context. Euskalingua 2005, V. 6, pp. 121-125. Disponível em: $<$ http://mendebalde.eus/euskalinguak/Euskalingua $\% 206 /$ Basque $\% 20$ textbooks $\% 20 \mathrm{during} \% 20$ the\%2020th\%20century.pdf>. Acesso em: 5 oct. 2015.

COLLELLDEMONT, E. (Coord). Investigar la Història de l'Educació amb imatges. Vic: Eumogràfic, 2014, 165p.

COMAS, F. y otros. Imatges de l'escola, imatges de l'educació. Palma de Mallorca: Universitat de les Illes Balears, 480p.

DÁVILA, P. El modelo histórico de alfabetización en Euskal Herria. In: Lengua, escuela y cultura. El proceso de alfabetización en Euskal Herria, siglos XIX y XX Leoia, Universidad del País Vasco, 1995, p. 17-44.

Euskal Herria tiene forma de corazón: la escuela en la construcción de la identidad nacional vasca. Historia de la educación. Revista Interuniversitaria, v. 27, p. 215-243, 2008.

DEL POZO, M ${ }^{\mathrm{a}}$ M. y RAMOS, S. Prácticas de escritura en los cuadernos escolares españoles (1920-1940). In: MEDA, J.; MONTINO, D. y SANI, R. School Exercice Books. A complex source for a History of the Approach to Schooling and Education in the $19^{\text {th }}$ and $20^{\text {th }}$ Centuries. Macerata, Univesita di Macerata, 2010, p. 823-845. 
DÍAZ NOCI, J. y otros. Jon Zabalo Ballarin “Txiki”. Donostia, Departamento de Cultura, Euskera, Juventud y Deportes. Diputación Foral de Gipuzkoa, 2003, 167p.

EUSKALTZAINDIA. EI libro blanco del euskara. Bilbao, Real Academia de la Lengua Vasca, 1977, 725p.

$214 p$.

Zipitria’tar Elbire “Andereñoa” Omenaldia. Tolosa, López Mendizabal, 1986,

FERNANDEZ, I. La escuela vasca y la larga historia de la postguerra. In DÁVILA, P. (ed.):

Lengua, escuela y cultura. EI proceso de alfabetización en Euskal Herria, siglos XIX y XX. Leoia, Universidad del País Vasco, 1995, p. 159-192.

FERNÁNDEZ, M. C.; WELTI, E. y BISELLI, R.: "La fuerza de la imagen en los cuadernos escolares de la Escuela Serena (Rosario, 1935-1950)" en MEDA, J.; MONTINO, D. y SANI, R.: Op. cit., p. 847-864.

GARMENDIA, M. Carmen y ETXEZARRETA, Iñaki: Ikastola. Auñamendi Eusko Entziklopedia, 2009, Disponível em: <http://www.euskomedia.org/aunamendi/ee73307/76041>. Acceso em: 15 jul. 2015.

MARTÍN, B. y RAMOS, I. Estudio y catálogo de cuadernos escolares. Cuadernos del CEMUPE. CEMUPE: Salamanca, 2013, 62p.

Escribir en la Escuela en tiempos de guerra. Bordón, v. 67, n 3, p. 67-84, 2015.

La Historia contada en los cuadernos escolares. Madrid, La Catarata, 2015, 176p.

MAYORDOMO, A. y FERNÁNDEZ, J. M. Vencer y convencer. Educación y política. España 1936-1945. Valencia, Universidad de Valencia, 1993, 208p.

MEDA, J. Los dibujos infantiles como fuentes históricas: perspectivas heurísticas y cuestiones metodológicas. Revista Brasileira de História da Educação, v. 14, n 3, p. 139$165,2014$.

O partigiano, portami via... La rappresentazione della Guerra di Liberazione nei componimenti scritti e nei disegni presentati dalle scuole italiane al Concorso nazionale sulla Resistenza (1965), History of Education and Children's Literature, v. 7, n.1, p. 261-294, 2012.

MEDA, J.; MONTINO, D. y SANI, R. School Exercice Books. A complex source for a History of the Approach to Schooling and Education in the $19^{\text {th }}$ and $20^{\text {th }}$ Centuries. Macerata, Univesita di Macerata, 2010, 1616p. 